\title{
Effects of calibration factors and intensity dependent non-linearity on functional photoacoustic microscopy
}

Amos Danielli, Junjie Yao, Arie Krumholz, Lihong V. Wang

Amos Danielli, Junjie Yao, Arie Krumholz, Lihong V. Wang, "Effects of calibration factors and intensity dependent non-linearity on functional photoacoustic microscopy," Proc. SPIE 7899, Photons Plus Ultrasound: Imaging and Sensing 2011, 78993C (28 February 2011); doi: $10.1117 / 12.875287$

SPIE. Event: SPIE BiOS, 2011, San Francisco, California, United States 


\title{
Effects of Calibration Factors and Intensity Dependent Non-linearity on Functional Photoacoustic Microscopy
}

\author{
Amos Danielli, Junjie Yao, Arie Krumholz, and Lihong V. Wang \\ Optical Imaging Laboratory, Department of Biomedical Engineering, Washington University in St. \\ Louis, MO 63130
}

\begin{abstract}
Functional photoacoustic microscopy is a valuable tool in quantifying hemoglobin oxygenation within single vessels. In several functional studies with this tool, quantitative $\mathrm{sO}_{2}$ measurements were taken both in vitro and in vivo. Although in vitro measurements of $\mathrm{sO}_{2}$ showed high agreement with expected values from premade samples, in practice, in vivo measurements were less accurate. The reported values of $70 \%-100 \% \mathrm{sO}_{2}$ in the arteries present large deviations from the expected range of $95-100 \%$. Several factors, such as fluence wavelength dependence, optical wavelength range, and transducer central frequency have been suggested and investigated in order to understand these discrepancies. Despite additional knowledge of systematic errors arising from such factors, measuring the absolute value of $\mathrm{sO}_{2}$ in vivo remains a challenge. All previous studies assumed linear dependence of the photoacoustic signal on absorption and used the linear least squares model. However, several factors, such as wavelength calibration errors, photodiode-wavelength dependence, and intensity dependent non-linearity, all of which may have a significant effect on the final calculation, have not been investigated. Here we evaluate both in vitro and in vivo the effects on $\mathrm{sO}_{2}$ measurements of photodiode wavelength dependence, laser wavelength accuracy, and intensity dependent absorption of oxygenated and deoxygenated hemoglobin. We show that these factors may contribute significantly to the deviations in $\mathrm{sO}_{2}$ calculations in vivo.
\end{abstract}

Keywords: Photoacoustic microscopy; non linear photoacoustic microscopy; oxygen saturation; optical saturation; photoacoustic tomography; photodiode calibration; wavelength calibration; OR-PAM;

\section{INTRODUCTION}

\subsection{Functional photoacoustic microscopy}

Photoacoustic microscopy and photoacoustic computed tomography are effective functional and molecular imaging tools in vivo. Because the photoacoustic (PA) signal is proportional to the deposited optical energy, which in turn is proportional to the local optical absorption coefficient, multi-wavelength photoacoustic measurements provide quantitative information about the concentrations of multiple chromophores, such as oxygenated and deoxygenated hemoglobin molecules in red blood cells. Thus, the relative concentration and the oxygen saturation $\left(\mathrm{sO}_{2}\right)$ of hemoglobin can be extracted [1-3]. Functional or molecular photoacoustic imaging of brain activation [2], tumor hemodynamics [4], gene expression $[5,6]$ and other pathophysiological phenomena has been previously achieved. Although in vitro measurements of $\mathrm{sO}_{2}$ showed high agreement with expected values from premade samples [7], in practice, in vivo measurements were less accurate. The reported values of $70 \%-100 \% \mathrm{sO}_{2}$ in the arteries $[8,9]$ present large deviations from the expected range of $95-100 \%$. Several factors, such as fluence wavelength dependence, optical wavelength range, and transducer central frequency have been suggested and investigated in order to understand these discrepancies. Other factors, such as wavelength calibration errors, photodiode-wavelength dependence, and intensity dependent nonlinearity, all of which may have a significant effect on the final calculation, have not been investigated. All prior quantitative functional photoacoustic studies have assumed a linear dependence between the photoacoustic signal and the local optical fluence and used the linear least squares model. The linear least-squares model relies on several assumptions that have to be verified. First, the absorption cross section and fluence are compensated accurately. Second, the photoacoustic signal is proportional to the incident fluence. Third, the Grueneisen parameter and the heat conversion efficiency are not wavelength dependant. The objective of this paper is to validate the assumptions and demonstrate the effect of discrepancies both in vitro and in vivo.

Photons Plus Ultrasound: Imaging and Sensing 2011, edited by Alexander A. Oraevsky, Lihong V. Wang, Proc. of SPIE Vol. 7899, 78993C · @ 2011 SPIE - CCC code: 1605-7422/11/\$18 - doi: 10.1117/12.875287 


\subsection{Theoretical model}

In an absorbing medium, the photoacoustic signal, $q$, generated by single photon absorption is proportional to the local pressure rise:

$$
q(F) \propto \Gamma \cdot \eta_{t h} \cdot \mu_{a} \cdot F,
$$

where $\Gamma$ is the Grueneisen parameter (dimensionless), $\eta_{t h}$ is the percentage of absorbed energy that is converted to heat, $\mu_{a}$ is the optical absorption coefficient $\left(\mathrm{cm}^{-1}\right)$, and $F$ is the local optical fluence $\left(\mathrm{J} / \mathrm{cm}^{2}\right)$. The local optical fluence can also be written as $F=I \cdot \tau_{\text {laser }}$, where $I$ is the local laser intensity $\left(\mathrm{W} / \mathrm{cm}^{2}\right)$ and $\tau_{\text {laser }}$ is the laser pulse width. The absorption coefficient of blood can be expressed as:

$$
\mu_{a}=\sigma_{H b R} \cdot N_{H b R}+\sigma_{H b O_{2}} \cdot N_{H b O_{2}},
$$

where $\sigma_{\mathrm{HbR}}$ and $\sigma_{\mathrm{HbO}_{2}}$ are the absorption cross sections of deoxyhemoglobin ( $\left.\mathrm{HbR}\right)$ and oxyhemoglobin $\left(\mathrm{HbO}_{2}\right)$ at a specific wavelength. $N_{H b R}$ and $\mathrm{N}_{\mathrm{HbO}_{2}}$ are the numbers of $\mathrm{HbR}$ and $\mathrm{HbO}_{2}$ absorbers, respectively, per unit volume. The linear equations linking the photoacoustic signal and the $\mathrm{HbR}$ and the $\mathrm{HbO}_{2}$ concentrations are

$$
\underbrace{\left[\begin{array}{c}
q\left(\lambda_{1}\right) / F\left(\lambda_{1}\right) \\
q\left(\lambda_{2}\right) / F\left(\lambda_{2}\right)
\end{array}\right]}_{\underline{y}}=\underbrace{\left[\begin{array}{ll}
\sigma_{\mathrm{HbO}_{2}}\left(\lambda_{1}\right) & \sigma_{H b R}\left(\lambda_{1}\right) \\
\sigma_{H_{b O}}\left(\lambda_{2}\right) & \sigma_{H b R}\left(\lambda_{2}\right)
\end{array}\right]}_{H} \times \underbrace{\left[\begin{array}{l}
\Gamma \cdot \eta_{t h} \cdot N_{H b O_{2}} \\
\Gamma \cdot \eta_{t h} \cdot N_{H b R}
\end{array}\right]}_{\underline{\theta}},
$$

Linear least-squares fitting with two measurements at different wavelengths recovers the relative concentrations of $\mathrm{HbR}$ and $\mathrm{HbO}_{2}$, and therefore the oxygen saturation in the vessel. The estimated parameters vector, $\underline{\hat{\theta}}$, is

$$
\underline{\hat{\theta}}=\left[\begin{array}{l}
\Gamma \cdot \eta_{t h} \cdot N_{H b O_{2}} \\
\Gamma \cdot \eta_{t h} \cdot N_{H b R}
\end{array}\right]=\left(H^{T} H\right)^{-1} H^{T} \times \underline{y} .
$$

For a $2 \times 2$ matrix, the estimated parameters vector is simply $\underline{\hat{\theta}}=H^{-1} \times y$. When the oxygen saturation is calculated in vivo, the vessel map may be segmented to allow averaging of the extracted concentrations of $\mathrm{HbO}_{2}$ and $\mathrm{HbR}$ molecules within the vessels. The $\mathrm{sO}_{2}$ is calculated as

$$
s \mathrm{O}_{2}=\frac{\left\langle\mathrm{N}_{\mathrm{HbO}}\right\rangle}{\left\langle\mathrm{H}_{\mathrm{HbO}}\right\rangle+\left\langle\mathrm{N}_{\mathrm{HbR}}\right\rangle},
$$

where $\langle\cdot\rangle$ denotes averaging. It should be noted that the linear least-squares solution consists of an inequality nonnegativity constraint (i.e., the solution for the estimated concentrations must be positive). When measuring the relative concentrations of oxyhemoglobin and deoxyhemoglobin molecules in the artery (where the concentration of the deoxyhemoglobin molecules is expected to be almost zero), measurement noise may produce negative estimations of the deoxyhemoglobin molecule concentrations. The inequality non-negativity constraint of the linear least-squares problem ensures non-negative concentrations of hemoglobin molecules.

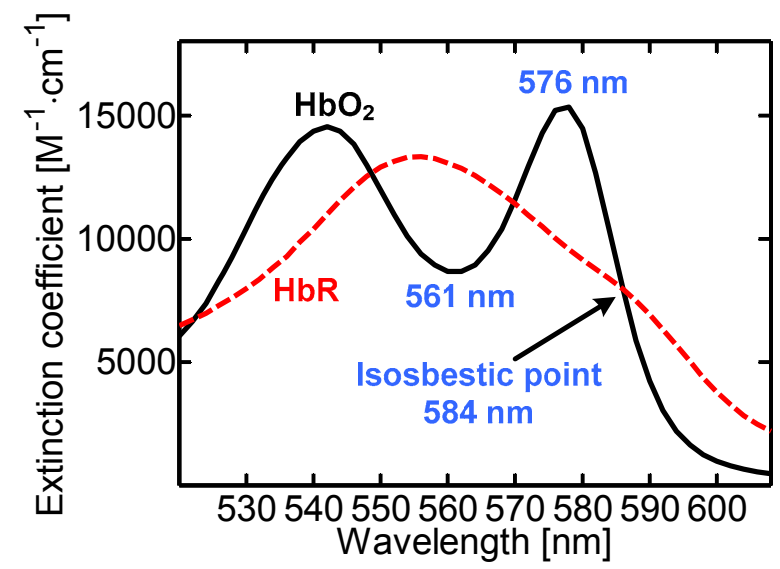

Figure 1: Molar extinction coefficient of oxyhemoglobin (black line) and deoxyhemoglobin (dashed red line) 


\section{EXPERIMENTAL SETUP AND RESULTS}

\subsection{Optical resolution PAM (OR-PAM)}

We measured the photoacoustic signal as a function of the incident laser fluence, using a custom-built OR-PAM system shown in Figure 2 . The 4 ns laser pulses are spatially filtered by a $50 \mathrm{~m}$ diameter pinhole and imaged by a microscope objective lens ( 0.1 N.A, Leica) to a focal spot with a diameter of $3.7 \mu \mathrm{m}$. A fraction of the fiber output light is reflected by a beam sampler and detected by a (photodiode S1226-18BK, Hamamatsu). The ultrasonic signal is collected by a concave lens and detected by a 50-MHz broadband ultrasonic transducer (V214BB, Panametrics). The optical objective lens and the ultrasonic transducer are coaxially and confocally aligned. For in vitro measurements, the sample holder is sealed with a plastic membrane and immersed in a water tank to provide acoustic coupling between the sample and the ultrasound transducer. We ensured the same beam diameter on the sample surface for all measurements by maintaining a constant delay time between the detected photoacoustic signal and the laser pulse. For in vivo measurements we imaged the ear of a Swiss-Webster mouse (25-30 g, Harlan).

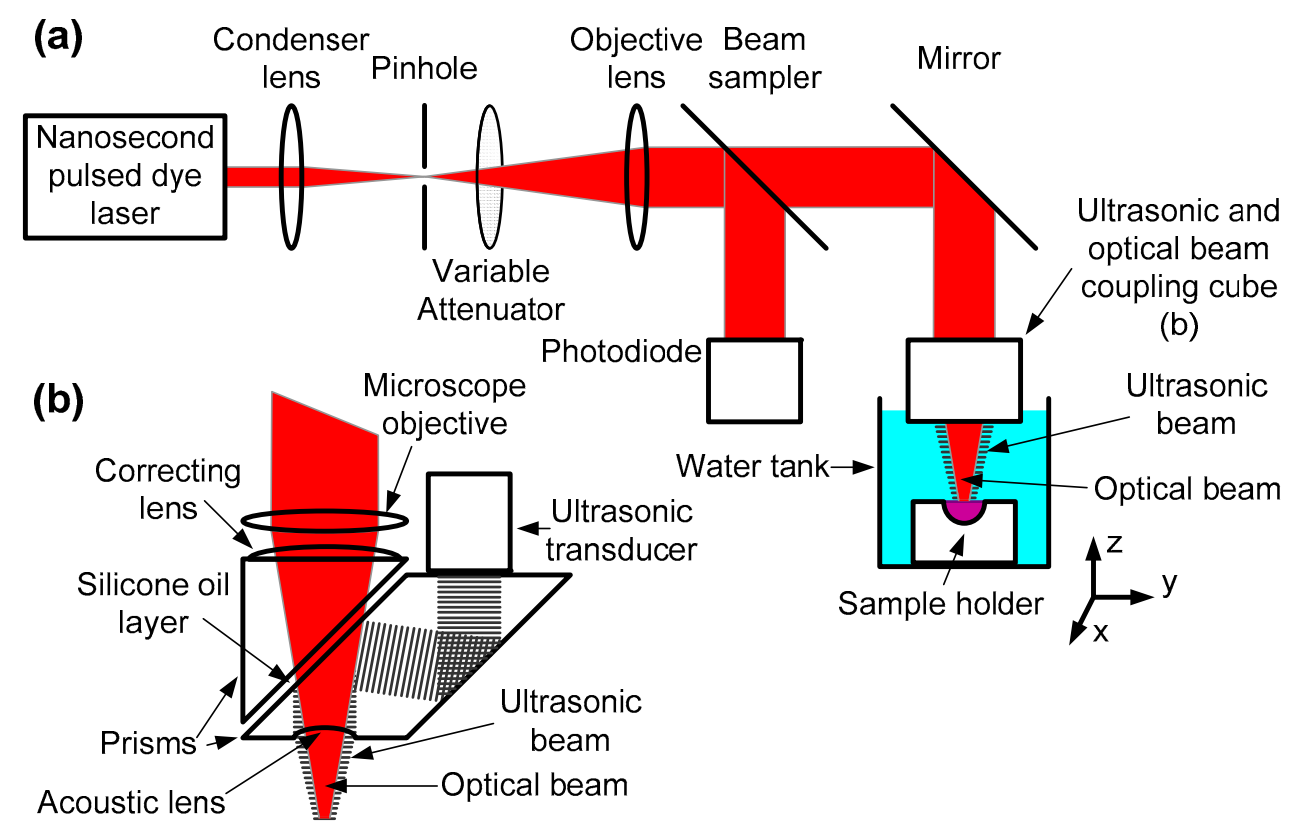

Figure 2: Schematic diagram of the Optical Resolution Photoacoustic Microscope (OR-PAM) system.

\subsection{Absorption cross section compensation}

The spectral region of interest for $\mathrm{sO}_{2}$ calculations used is between 560 and $600 \mathrm{~nm}$. The molar extinction coefficients (and the subsequent cross sections) of $\mathrm{HbO}_{2}$ and $\mathrm{HbR}$ are taken from the published data to generate the coefficients matrix $(\mathrm{H})$. Uncertainty in the laser wavelength leads to shifted values of the absorption cross sections, and therefore different solution to the linear least squares fitting. The error is maximal when the coefficient matrix is approximately singular. The singularity of the matrix implies that any uncertainty in the excitation laser (i.e., modified cross sections) will greatly affect the estimated concentrations of oxy and deoxyhemoglobin. Figure 3 presents the determinant of the extinction coefficients matrix using wavelength pairs with $6 \mathrm{~nm}$ difference. When one of the wavelengths is near an isosbestic point the determinant is non-zero and the linear least squares algorithm has an invertible matrix. In this case, the matrix is less susceptible to wavelength inaccuracy, i.e., minor variation in the extinction coefficient values will not change much the inverted matrix. However, for some wavelength pairs (e.g., $576 \mathrm{~nm}$ and $582 \mathrm{~nm}$ ), the determinant is close to zero and therefore, the matrix is near singularity. Any wavelength inaccuracy will greatly affect the estimation of the concentrations. To evaluate the effects of wavelength inaccuracy, we examine the case of fully oxygenated blood (e.g., at the artery, the oxygen saturation is near $100 \%$ ). In this case, Eq. 3 can be reduced to: 


$$
\left[\begin{array}{c}
q\left(\lambda_{1}\right) / F\left(\lambda_{1}\right) \\
q\left(\lambda_{2}\right) / F\left(\lambda_{2}\right)
\end{array}\right]=\left[\begin{array}{ll}
\sigma_{\mathrm{HbO}_{2}}\left(\lambda_{1}\right) & \sigma_{H b R}\left(\lambda_{1}\right) \\
\sigma_{\mathrm{HbO}_{2}}\left(\lambda_{2}\right) & \sigma_{H b R}\left(\lambda_{2}\right)
\end{array}\right] \times\left[\begin{array}{c}
\Gamma \cdot \eta_{t h} \cdot N_{\mathrm{HbO}_{2}} \\
\sim 0
\end{array}\right]=\left[\begin{array}{l}
\sigma_{\mathrm{Hbo}_{2}}\left(\lambda_{1}\right) \cdot \Gamma \cdot \eta_{t h} \cdot N_{H b O_{2}} \\
\sigma_{\mathrm{HbO}_{2}}\left(\lambda_{2}\right) \cdot \Gamma \cdot \eta_{t h} \cdot N_{H b O_{2}}
\end{array}\right] .
$$

Therefore, the ratio between the photoacoustic signals (normalized by the fluence) at different wavelengths should be the ratio between the cross sections of oxyhemoglobin at these wavelengths. For example, when the wavelength pair is 584 $\mathrm{nm}$ and $590 \mathrm{~nm}$, the ratio between the cross sections for bovine blood is expected to be [10]: $\sigma_{\mathrm{HbO}_{2}}(584) / \sigma_{\mathrm{HbO}_{2}}(590)=2.44$. However, wavelength inaccuracy of $0.5 \mathrm{~nm}$ results with a different ratio $\sigma_{\mathrm{HbO}_{2}}(583.5) / \sigma_{\mathrm{HbO}_{2}}(589.5)=2.35$. Thus, the extracted concentrations will deviate from their expected values and the calculated oxygen saturation will be reduced from $100 \%$ to $\sim 95.5 \%$ ( $4.5 \%$ error).

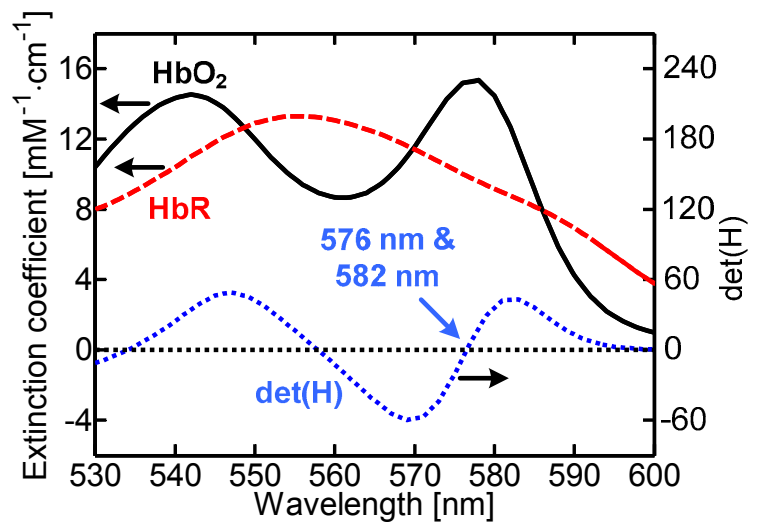

Figure 3: The determinant of the extinction coefficients matrix (dashed blue line) using wavelength pairs with $6 \mathrm{~nm}$ difference.

\subsection{Fluence compensation}

Fluence compensation can be achieved using the photodiode (PD) readout. The photodiode voltage is known to have wavelength dependence (approximately $1 \%$ change per $10 \mathrm{~nm}$ according to the photodiode's manufacturer specification). However, the photodiode voltage as a function of the laser pulse energy presented much higher wavelength dependence than expected (approximately 6\% change per $10 \mathrm{~nm}$ ). This calibration takes into account all optical parts (beam sampler and photodiode).

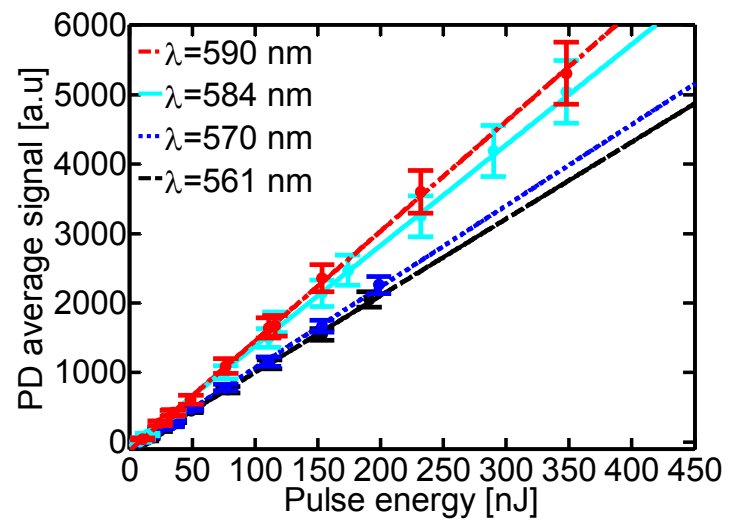

Figure 4: Photodiode readout as a function of the OR-PAM output power for different wavelengths

Without photodiode calibration, the fluence is taken to be the same for different wavelengths assuming the photodiode readout is equal. The photodiode calibration shows that for a given photodiode readout, the actual pulse energy is different for different wavelengths. For example, for the wavelengths pair $584 \mathrm{~nm}$ and $590 \mathrm{~nm}$, the average difference is 
$\sim 5 \%$. When imaging a fully oxygenated blood sample, neglecting this difference reduces the oxygen saturation calculation from $100 \%$ to $94 \%$ ( $6 \%$ error).

\subsection{Non-linear photoacoustic microscopy}

The optical-resolution photoacoustic microscopy (OR-PAM) was developed to achieve higher resolution by reducing the laser beam diameter, which increases the light intensity. As intensity increases, mechanisms such as saturation of the optical absorption or multi-photon/multi-step absorption can occur, resulting in a nonlinear dependence of the photoacoustic signal on the excitation pulse fluence [11-13]. The photoacoustic signal, $q$, can be written as:

$$
q(F) \propto \Gamma \cdot \eta_{t h} \cdot \sigma \cdot \frac{N_{0}}{1+\frac{I}{I_{\text {sat }}}} \cdot F+k_{2} \cdot F^{2}
$$

where $N_{0}$ is the number of absorbers per unit volume, $I_{\text {sat }}$ is the saturation intensity, and $k_{2}$ is the two-photon / multistep absorption coefficient. The saturation intensity is a property of the absorber and it is given by

$$
I_{\text {sat }}=\frac{h \cdot v}{\sigma \cdot \tau_{e f f}}
$$

where $h$ is Plank's constant, $v$ is the laser frequency, and $\tau_{e f f}$ is the absorption relaxation time (i.e., the time required for an excited molecule to relax to the ground state after light absorption). The saturation intensity is at minimum when the absorption cross section is at maximum. Therefore, the photoacoustic signal saturates with lower laser intensity at wavelengths near the absorption peak of the absorber. Moreover, the saturation intensity depends on the absorption relaxation time and cross section. For example, oxyhemoglobin and deoxyhemoglobin exhibit different saturation curves at $576 \mathrm{~nm}$, the local absorption peak of $\mathrm{HbO}_{2}$ (see Figure 5 [11]).

(a)

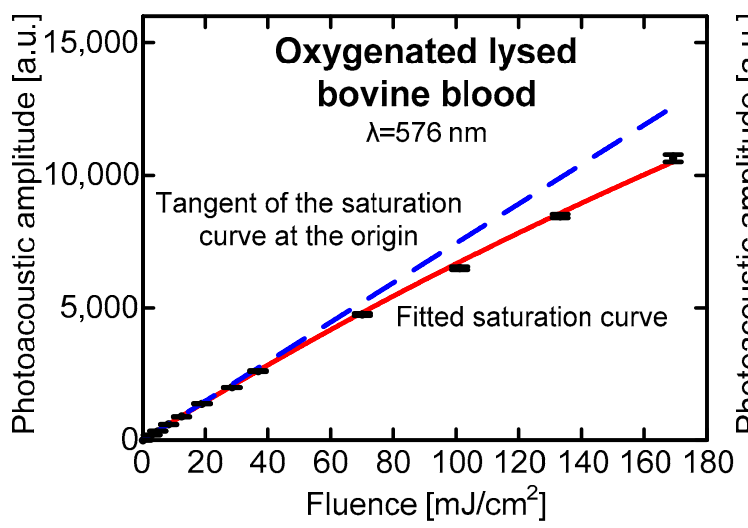

(b)

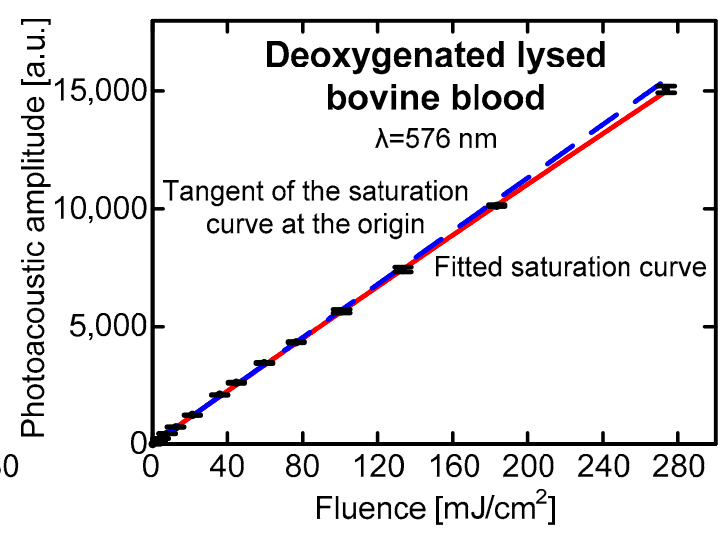

Figure 5: The photoacoustic signal as a function of the incident fluence at $576 \mathrm{~nm}$ on (a) oxygenated lysed bovine blood and (b) deoxygenated lysed bovine blood

In addition to the optical non linearity, when the temperature rise due to the energy deposition is high there can be temperature non linearity. The Grueneisen parameter, which depends on the temperature $T$, changes in the form

$$
\Gamma(\mathrm{T}) \cong \Gamma\left(\mathrm{T}_{0}\right)+\frac{\partial \Gamma}{\partial T} \cdot \Delta T
$$

where $\Delta T \propto \mu_{a} \cdot F$ is the temperature rise. The temperature non linearity adds to the parabolic dependence of the photoacoustic signal on the fluence. When the photoacoustic signal is not proportional to the fluence, the linear leastsquares algorithm is not valid and the calculations of $\mathrm{sO}_{2}$ are incorrect.

\subsection{In vitro photoacoustic microscopy}

We measured the photoacoustic signal (maximum peak to peak amplitude) from $\mathrm{HbO}_{2}$ and $\mathrm{HbR}$ as a function of the incident pulse energy at $584 \mathrm{~nm}$ and $590 \mathrm{~nm}$. Oxygenated lysed bovine blood (50:50 DI-water and defibrinated bovine blood) was purchased from Quadfive (905-250). The oxygenated level was measured to be $99 \%$ using a spectrophotometer. Deoxygenated hemoglobin was prepared by mixing carbon dioxide with defibrinated bovine blood (910-250, Quadfive) for 2 hours at $37^{\circ} \mathrm{C}$. The oxygenated level was measured to be $32 \pm 1$ using a gas analyzer (Stat Profile ${ }^{\circledR}$ pHOx ${ }^{\circledR}$ Plus, Nove biomedical). The results are presented in Figure 6. While the PA signal from the deoxygenated sample showed a linear dependence with the laser pulse energy (Figure 6a), the signal from the oxygenated sample started to show a parabolic dependence with increasing incident energy (Figure 6b). To reduce any 
temperature non-linearity, we repeated the experiment using a diluted oxygenated lysed blood (1:4 oxygenated lysed blood and DI water). The absorption coefficient of the diluted sample (and consequently, the temperature rise) is reduced by a factor of 4 . However, the PA signal from the diluted oxygenated lysed blood showed similar dependence (Figure $6 \mathrm{c}$ ), suggesting that the temperature rise is not the dominant process. Moreover, the temperature rise of the deoxygenated bovine blood was much higher than that of the diluted oxygenated bovine blood and yet it didn't demonstrate a parabolic dependence for the same incident energy levels.
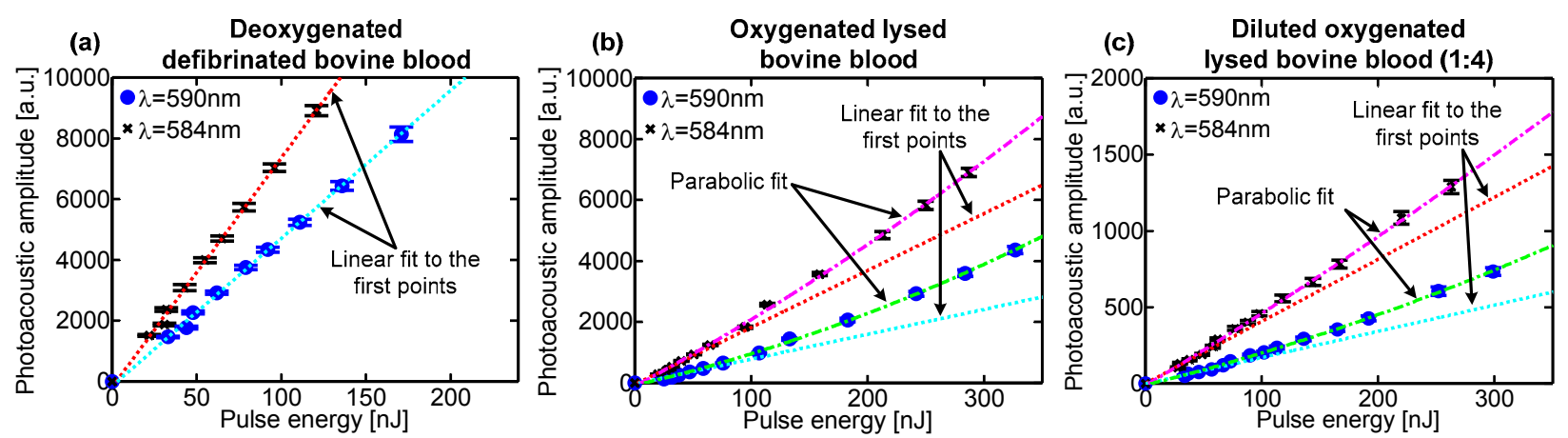

Figure 6: The photoacoustic signal as a function of the incident pulse energy at $584 \mathrm{~nm}$ and $590 \mathrm{~nm}$ from (a) deoxygenated defibrinated bovine blood ( $32 \pm 1 \%$ oxygen saturation), (b) oxygenated lysed bovine blood ( $99 \%$ oxygen saturation), and (c) diluted oxygenated lysed bovine blood ( $99 \%$ oxygen saturation)

We extracted the relative concentrations of oxyhemoglobin and deoxyhemoglobin molecules using the linear leastsquares algorithm and calculated the oxygen saturation (Figure 7). The oxygen saturation of the oxygenated sample was calculated to be $98.4 \% \pm 1$ at low pulse energies. As the pulse energy increases, the calculated $\mathrm{sO}_{2}$ decreased with an increasing error. The oxygen saturation from the deoxygenated sample was relatively constant with increasing pulse energies $(37 \pm 2 \%)$. At low pulse energy levels, the ratio between the cross sections of oxygenated bovine blood at 584 $\mathrm{nm}$ and $590 \mathrm{~nm}$ matches the ratio between the slopes of the PA signal curves at these wavelengths (approximately 2.44 , Figure 6b). However, as the pulse energy increases, the slope of the PA signal curve at $590 \mathrm{~nm}$ increases faster than the slope of the PA signal curve at $584 \mathrm{~nm}$. Thus, the ratio between the slopes and subsequently, the calculated $\mathrm{sO}_{2}$, decreases. The average decrease in the ratio of the PA signal slopes at $584 \mathrm{~nm}$ and $590 \mathrm{~nm}$ is estimated to be 13\% (for laser pulse energies between $50 \mathrm{~nJ}$ to $150 \mathrm{~nJ}$ ).

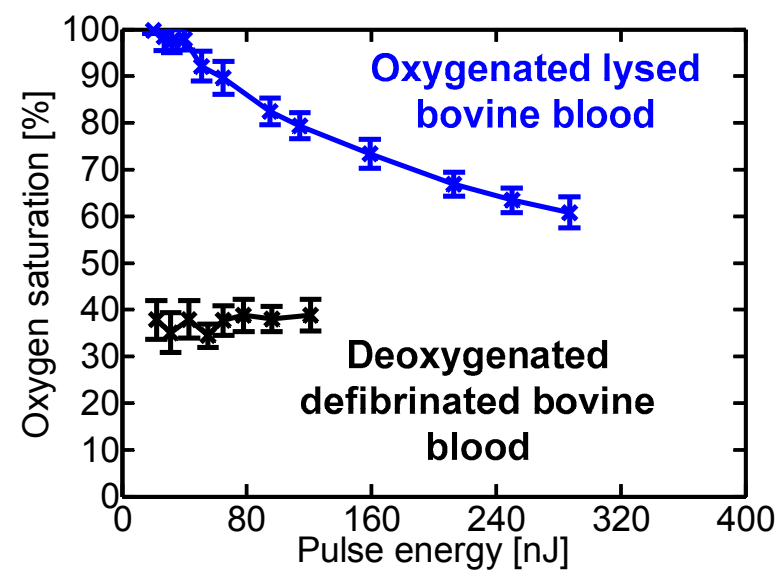

Figure 7: oxygen saturation calculation of oxygenated lysed bovine blood ( $99 \%$ oxygen saturation measured with spectrophotometer) and deoxygenated defibrinated bovine blood ( $32 \pm 1 \%$ oxygen saturation measured with a gas analyzer)

\subsection{In vivo photoacoustic microscopy}

First, we calibrated our photodiode readout over a wide range of energy levels and measured the laser wavelength using a wavelength meter (WaveMaster Wavelength Meter, 33-2650-00, Coherent). Then, we imaged the same area of the mouse ear at nine different intensities using the wavelength pair $584 \mathrm{~nm}$ and $590 \mathrm{~nm}$. Due to the low signal to noise ratio 
of the PA signal, we had to use energy levels above $50 \mathrm{~nJ}$. Therefore, at each intensity level, we multiplied the PA signal generated at wavelength $584 \mathrm{~nm}$ by $13 \%$ to correct for the expected non-linearity. We then extracted the relative concentrations of oxyhemoglobin and deoxyhemoglobin at each pixel and calculated the oxygen saturation. Figure 8a presents the average oxygen saturation of the artery and the vein as a function of the incident pulse energies. The average $\mathrm{sO}_{2}$ at the artery and the vein for incident pulse energies between $70 \mathrm{~nJ}$ and $150 \mathrm{~nJ}$ was calculated to be $95 \pm 1 \%$ and $71 \pm 2 \%$, respectively. As the incident pulse energies increase, the calculated $\mathrm{sO}_{2}$ decreases due to the non linearity of the oxyhemoglobin photoacoustic signal.
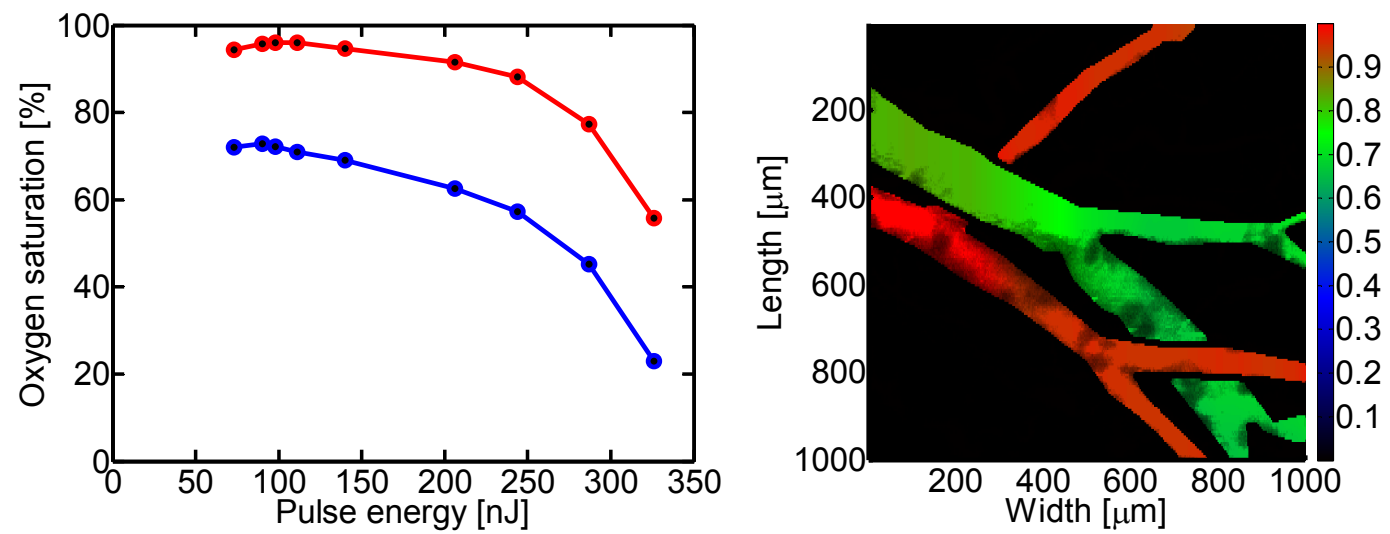

Figure 8: (a) Average oxygen saturation at the artery and the vein as a function of the incident pulse energies (b) Oxygen saturation map of mouse ear vessels

\section{CONCLUSSIONS}

In conclusion, we showed that factors such as wavelength calibration errors and photodiode-wavelength dependence may significantly affect the $\mathrm{sO}_{2}$ calculation. Moreover, in vitro measurements of the PA signal from oxyhemoglobin and deoxyhemoglobin showed deviation from linearity, which further affect the $\mathrm{sO}_{2}$ calculations. Oxyhemoglobin has lower saturation intensity than deoxyhemoglobin, particularly at $576 \mathrm{~nm}$, the local absorption peak of oxyhemoglobin. We showed that at high intensity and away from the local absorption peak (at $584 \mathrm{~nm}$ and $590 \mathrm{~nm}$ ) the PA signal from oxyhemoglobin is influenced by other mechanisms, such as two-photon / multi-step excitation. However, the PA signal from deoxyhemoglobin at these wavelengths was influenced by a single photon absorption only, yielding a linear dependence on the laser fluence even at high fluence. We evaluated the error induced by the oxyhemoglobin nonlinearity at these wavelengths and used it to correct the in vivo measurements of the oxygen saturation in a mouse ear.

\section{REFERENCES}

[1] Laufer, J. G., Delpy, D., Elwell, C., and Beard, P. C., "Quantitative spatially resolved measurement of tissue chromophore concentrations using photoacoustic spectroscopy: application to the measurement of blood oxygenation and haemoglobin concentration," Phys. Med. Biol. 52, 141-168 (2007).

[2] Wang, X., Xie, X., Ku, G., and Wang, L. V., "Noninvasive imaging of hemoglobin concentration and oxygenation in the rat brain using high-resolution photoacoustic tomography," J. Biomed. Opt. 11, 024015 (2006).

[3] Zhang, H. F., Maslov, K., Stoica, G., and Wang, L. V., "Functional photoacoustic microscopy for high-resolution and noninvasive in vivo imaging," Nat. Biotechnol. 24, 848-851 (2006).

[4] Kim, C., Favazza, C., and Wang, L. V., "In Vivo Photoacoustic Tomography of Chemicals: High-Resolution Functional and Molecular Optical Imaging at New Depths," Chem. Rev. , Vol. 110(5), pp. 2756-2782 (2010).

[5] Razansky, D., Distel, M., Vinegoni, C., Ma, R., Perrimon, N., Koster, R. W., and Ntziachristos, V., "Multispectral opto-acoustic tomography of deep-seated fluorescent proteins in vivo," Nat. Photonics, 3, $412-417$ (2009)

[6] Li, L., Zemp, R., Lungu, G., Stoica, G., and Wang, L. V., "Photoacoustic imaging of lacZ gene expression in vivo," J. Biomed. Opt 12(02), 020504 (2007)

[7] Zhang, H. F., Maslov, K., Sivaramakrishnan, M., Stoica, G., and Wang, L. V., "Imaging of hemoglobin oxygen saturation variations in single vessels in vivo using photoacoustic microscopy," Appl. Phys. Lett. 90(5), 053901 (2007) 
[8] Maslov, K., Zhang, H. F., and Wang, L. V., "Effects of wavelength-dependent fluence attenuation on the noninvasive photoacoustic imaging of hemoglobin oxygen saturation in subcutaneous vasculature in vivo," Inverse Probl. 23, 113-122 (2007).

[9] Sivaramakrishnan, M., Maslov, K., Zhang, H. F., Stoica, G., and Wang, L. V., "Limitations of quantitative photoacoustic measurements of blood oxygenation in small vessels," Phys. Med. Biol. 52(5), 1349-1361 (Mar. 2007)

[10]Zijlstra, W. G., Buursma, A., and van Assendelft, O. W., [Visible and Near Infrared Absorption Spectra of Human and Animal Hemoglobin, Determination and Application], VSP, Amsterdam The Netherlands, 103-132 (2000)

[11] Danielli, A., Favazza, C. P., Maslov, K., and Wang, L. V., "Picosecond absorption relaxation measured with nanosecond laser photoacoustics," Appl. Phys. Lett. 97, 163701 (2010)

[12] Tam, A. C., and Patel, C. K. N., "Two-photon absorption spectra and cross-section measurements in liquids," Nature 280, 304-306 (1979).

[13] Sathy, P., Philip, R., Nampoori, V. P. N., and Vallabhan, C. P. G., "Photoacoustic observation of excited singlet state absorption in the laser dye Rhodamine 6G," J. Phys. D Appl. Phys. 27, 2019-2022 (1994) 